\title{
The global mass function of M 15
}

\author{
A. Pasquali ${ }^{1, \star}$, G. De Marchi ${ }^{2, \star \star}$, L. Pulone ${ }^{3}$, and M. S. Brigas ${ }^{1,4}$ \\ ${ }^{1}$ ESO/ST-ECF, Karl-Schwarzschild-Strasse 2, 85748 Garching bei München, Germany \\ e-mail: apasqual@eso.org \\ 2 ESA, Space Telescope Operations Division, 3700 San Martin Drive, Baltimore, MD 21218, USA \\ e-mail: gdemarchi@rssd.esa.int \\ 3 INAF, Osservatorio Astronomico di Roma, Via di Frascati 33, 00040 Monte Porzio Catone (RM), Italy \\ e-mail: pulone@coma.mporzio.astro.it \\ 4 Osservatorio Astronomico di Cagliari, Strada 54, Poggio dei Pini, 09012 Capoterra, Cagliari, Italy \\ e-mail: sbrigas@eso.org
}

Received 14 August 2003 / Accepted 21 July 2004

\begin{abstract}
Data obtained with the NICMOS instrument on board the Hubble Space Telescope (HST) have been used to determine the $H$-band luminosity function (LF) and mass function (MF) of three stellar fields in the globular cluster M 15, located $\sim 7^{\prime}$ from the cluster centre. The data confirm that the cluster MF has a characteristic mass of $\sim 0.3 M_{\odot}$, as obtained by Paresce \& De Marchi (2000) for a stellar field at 4.6 from the centre. By combining the present data with those published by other authors for various radial distances (near the centre, at $20^{\prime \prime}$ and at 4.6), we have studied the radial variation of the LF due to the effects of mass segregation and derived the global mass function (GMF) using the Michie-King approach. The model that simultaneously best fits the LF at various locations, the surface brightness profile and the velocity dispersion profile suggest that the GMF should resemble a segmented power-law with the following indices: $x \simeq 0.8$ for stars more massive than $0.8 M_{\odot}, x \simeq 0.9$ for $0.3-0.8 M_{\odot}$ and $x \simeq-2.2$ at smaller masses (Salpeter's IMF would have $x=1.35$ ). The best fitting model also suggests that the cluster mass is $\sim 5.4 \times 10^{5} M_{\odot}$ and that the mass-to-light ratio is on average $M / L_{V} \simeq 2.1$, with $M / L_{V} \simeq 3.7$ in the core. A large amount of mass $(\sim 44 \%)$ is found in the cluster core in the form of stellar heavy remnants, which may be sufficient to explain the mass segregation in M 15 without invoking the presence of an intermediate-mass black hole.
\end{abstract}

Key words. globular clusters: general - globular clusters: individual: M 15

\section{Introduction}

M 15 (NGC 7078) is a classical target for studying the internal dynamics of globular clusters from the observed surface brightness and velocity dispersion profiles for it is at a relatively large distance from the Galactic plane $\left(Z_{\mathrm{G}}=-4.7 \mathrm{kpc}\right.$; Harris 1996). This location, combined with an orbit of small ellipticity ( $e=0.34$; Dinescu et al. 1999), minimises the cluster interaction with the Galaxy and hence tidal stripping and evaporation of stars from the cluster outskirts. Ground-based observations revealed the presence of a central cusp which has been attributed to core collapse (Djorgovski \& King 1986). Recent HST observations have not been able to clarify the nature of the M 15 cusp, in spite of their enhanced spatial resolution. The observed stellar density profile can in fact be reproduced by assuming the existence of either a central black hole (Guhathakurta et al. 1996) or a compact core as the byproduct

^ Present address: Institut für Astronomie, ETH Hoenggerberg, CH-8093 Zürich, Switzerland.

$\star \star$ Present address: ESA, Science Communication Office, Keplerlaan 1, 2200 AG Noordwijk, The Netherlands. of the cluster core collapse in the presence of diffuse dark matter (Lauer et al. 1991). Very recently, Baumgardt et al. (2002) and Gerssen et al. (2002) have interpreted the kinematical data obtained with STIS and WFPC2 as due to either strong segregation of stellar remnants (white dwarfs and neutron stars, as already suggested by Illingworth \& King 1977) or to the presence of a $\sim 10^{3} M_{\odot}$ black hole in the core of M 15. As before, both explanations are statistically equivalent.

Further evidence of mass segregation in the central regions of M 15 comes from the detection of colour gradients whereby $(U-B)$ and $(B-V)$ colours get bluer towards the cluster centre (Bailyn et al. 1989; Cederbloom et al. 1992). They have been justified in terms of either a core concentration of blue stars due to binary-single star interactions or the central lack of low mass main-sequence stars. In either case, mass segregation is likely to be the driving mechanism. De Marchi \& Paresce (1994) have resolved with HST/FOC a large number of bright blue stars in the core of M 15 , the majority of which can be classified as blue stragglers. Nevertheless, the rest appear to belong to a new, as yet unidentified class of very blue stars. Amongst several possibilities (such as Early-Post AGB, subdwarfs and 
Table 1. Log of the observations.

\begin{tabular}{llcccccc}
\hline \hline & Dataset & $\begin{array}{c}\text { RA (h) } \\
J=2000\end{array}$ & $\begin{array}{c}\text { Dec (d) } \\
J=2000\end{array}$ & $\begin{array}{c}\text { PA (d) of the } \\
\text { detector } Y \text { axis }\end{array}$ & Filter & $\begin{array}{c}\text { Number of } \\
\text { images }\end{array}$ & $\begin{array}{c}\text { Total exposure } \\
\text { time (s) }\end{array}$ \\
\hline \multirow{2}{*}{ Field 1 } & n4k6r0hca & $21: 30: 17.22$ & $12: 15: 55.4$ & 0.341 & $F 110 W$ & 4 & 767.835 \\
& n4k6r0hda & $21: 30: 17.22$ & $12: 15: 55.4$ & 0.341 & $F 160 W$ & 4 & 1023.823 \\
Field 2 & n4k6r2hsa & $21: 30: 19.01$ & $12: 15: 51.5$ & 0.342 & $F 110 W$ & 14 & 2687.426 \\
& n4k6r2hta & $21: 30: 19.01$ & $12: 15: 51.5$ & 0.342 & $F 160 W$ & 14 & 3583.384 \\
Field 3 & n4k6u0v8a & $21: 30: 15.10$ & $12: 16: 34.2$ & -8.546 & $F 110 W$ & 18 & 3455.262 \\
& n4k6u0v9a & $21: 30: 15.10$ & $12: 16: 34.2$ & -8.546 & $F 160 W$ & 18 & 4607.208 \\
\hline
\end{tabular}

well-mixed single stars), De Marchi \& Paresce (1994, 1996) have suggested that dynamical interactions and close encounters could have stripped off the envelope of red giant stars, enhancing their mass loss and heading their evolution towards the late stage of helium white dwarf and $\mathrm{CO}$ white dwarf.

Because of minimal interactions with the Galaxy, the outskirts of M 15 have been observed to constrain the cluster initial MF (IMF) of low mass stars. For example, De Marchi \& Paresce (1995) performed deep HST/WFPC2 photometry of a field 4.6 NW of the centre and derived the Luminosity Function (LF) of main-sequence stars down to $M_{I} \simeq 10$, or two magnitudes fainter than the LF peak. The mass distribution that Paresce \& De Marchi (2000) have subsequently inferred from this LF shows that the characteristic mass of M 15 is $\sim 0.30 M_{\odot}$ and that the number of stars less massive than $0.3 M_{\odot}$ quickly drops off. This appears to be a common feature of all Galactic globular clusters for which deep LFs are available, regardless as to their metallicity, position in the Galaxy and dynamics (Piotto et al. 1997; Paresce \& De Marchi 2000). This MF is believed to be representative of the IMF of globular clusters, however the true IMF can be reliably established only by disentangling the cluster dynamical evolution from the observed MF. This is best achieved when MFs are available at several distances from the cluster centre. For M 15, MFs are found in the literature near the core (De Marchi \& Paresce 1996; Sosin \& King 1997) and for the above mentioned field at 4.6 NW of the cluster centre. Deep images taken by HST/NICMOS Camera 3 (NIC3) during the 1998 parallel campaign have allowed us to derive the MF in three, outer fields at $7^{\prime} \mathrm{NE}$ of the centre. We have used this mass distribution together with those published previously to constrain the Global Mass Function (GMF) of M 15. If the interaction of M 15 with the Galactic tidal field has been as weak as recent works indicate (Gnedin \& Ostriker 1997; Dauphole et al. 1996), then the GMF should reflect the IMF.

The NIC3 observations are presented in Sect. 2 and the data reduction is described in Sect. 3. The LF of the NIC3 fields in the $H$ band and its corresponding MF are discussed in Sect. 4 and compared with other literature measurements in Sect. 5. We derive the GMF in Sect. 6 and our conclusions follow in Sect. 7.

\section{Observations}

M 15 has been observed with the NIC3 camera of HST/NICMOS on 1998 July 7th and 18th, during the parallel observations campaign. Three overlapping fields have been imaged at about $7^{\prime} \mathrm{NE}$ from the centre of the cluster, at a distance of 7 times the half-light radius (1'; Trager et al. 1995). Multiple exposures have been taken of each field through both the $F 110 \mathrm{~W}$ and $F 160 \mathrm{~W}$ filters, centered at $1.1 \mu \mathrm{m}$ and $1.6 \mu \mathrm{m}$, respectively. The coordinates of the fields and the names, filters, and total exposure times of the image data-sets are given in Table 1 . Hereafter we refer to the $F 110 \mathrm{~W}$ and $F 160 \mathrm{~W}$ bands as $J$ and $H$, respectively.

\section{Data reduction}

The images were reduced using the NICMOS standard calibration pipeline: they were first processed with CALNICA for bias subtraction, dark-count correction and flat-fielding. Images belonging to the same field were then associated by means of the IRAF routine MAKEASSOCIATION and combined with CALNICB, to remove cosmic rays and to increase the signalto-noise ratio.

Photometry was performed on each of the three combined images with the DAOPHOT package. Stars were identified with DAOFIND, by setting the detection threshold at $5 \sigma$ above the local background. We traced the radial profile of each identified object and discarded those with full width at half maximum $(F W H M)$ larger than 2.5 pixels, since the typical $F W H M$ of a well defined point source in our frames is 1.5 pixels. Moreover, we compared images of the same association in order to identify bad pixels not flagged by the calibration pipeline. In this way we selected a sample of 539 stars imaged in both $J$ and $H$ bands. Because of the highly variable background, we decided to measure stellar count-rates in small fixed apertures of 2 pixels in radius (equivalent to 0 .' $^{\prime}$ ), and the corresponding background values in a fixed annulus with a radius of 5 pixels and a width of 2 pixels. After background subtraction and before applying any aperture correction, we corrected the countrates for the NIC3 intra-pixel sensitivity, using the equations computed by Storrs et al. (1999; see Table 2) in the case of out-of-focus campaign data.

The aperture correction was determined in three steps:

i) First, we constructed a mean growth curve for each frame from a sample of bright and isolated stars. The stellar fluxes were measured in 10 apertures, with radii ranging from 1 through to 7 pixels, and the sky was taken in a fixed annulus with a radius of 7 pixels and a width of 3 pixels. 
Table 2. Estimated photometric errors.

\begin{tabular}{lcc}
\hline \hline Bin & Error in $H$ & Error in $J$ \\
\hline mag. $\leq 22$ & 0.05 & 0.05 \\
$22<$ mag. $\leq 23$ & 0.10 & 0.09 \\
mag. $>23$ & 0.12 & 0.21 \\
\hline
\end{tabular}

After background subtraction, the stellar count-rates obtained for the same aperture were averaged into a mean growth curve, from which we derived the amount of energy encircled between 2 and 5 pixels, needed to scale our count rates to an aperture of 5 pixels.

ii) Since NIC3 was out of focus during our observations, we used the TinyTim software (Krist \& Hook 1999) to simulate the instrumental point spread function (PSF) with the precise optics settings corresponding to a specific filter and observation date. We computed two PSFs for each frame, one for our observation date (July 1998) and one for 1998 January 15, when NIC3 was in-focus (in-focus campaigns were carried out in January and June 1998). We calculated the encircled energy for a 5 pixels aperture for each PSF and used the flux ratio of in-focus and out-offocus to correct our measured count rates.

iii) We finally multiplied the sample count rates by the factor of 1.075 so as to correct them to the values measured in a nominal infinite aperture (NICMOS Photometry Cookbook, cf. http://www.stsci.edu/ hst/nicmos/performance).

The corrected count rates $c$ were then converted to magnitudes in the VEGAMAG photometric system by means of the relation:

$m=-2.5 \log \left(\frac{c U}{Z}\right)$

where $U$ is the conversion factor from flux to count rate and $Z$ is the flux for a zero magnitude star in the VEGAMAG system, provided for all NICMOS filters and VEGAMAG bands by the HST Data Handbook (http://www.stsci.edu/hst/nicmos/documents/ handbooks).

\subsection{Photometric uncertainty}

We estimated the internal uncertainty of our photometry by comparing the resulting magnitudes of those stars in common to two different fields: Fields 1 and 2 overlap nearly over two quadrants sharing 83 stars, whilst Fields 1 and 3 have in common only 12 stars. As a measure of the uncertainty, we used the difference between the magnitudes measured in each field and the weighted mean of the two values, with the weight given by the square root of the exposure time. The resulting errors, for Field 2, are shown in Table 2 for three magnitude ranges. We associate an uncertainty of 0.05 mag to stars brighter than magnitude 22 and a photometric error $>0.1$ to fainter objects. As regards Field 1, errors are usually twice as large, since the exposure time is $\sim 3.5$ times shorter in both bands. For Field 3 we adopted the same uncertainty scale as for Field 2.
Table 3. Completeness factors.

\begin{tabular}{lccc}
\hline \hline Field & bin & $f(J$ band $)$ & $f(H$ band $)$ \\
\hline 1 & $15-22$ & 1. & 1. \\
2 & & 1. & 1. \\
3 & & 1. & 1. \\
\hline 1 & $22-23$ & 0.959 & 0.808 \\
2 & & 0.949 & 0.935 \\
3 & & 0.913 & 0.864 \\
\hline 1 & $23-23.5$ & 0.807 & 0.526 \\
2 & & 0.935 & 0.821 \\
3 & & 0.909 & 0.680 \\
\hline 1 & $23.5-24$ & 0.667 & 0.274 \\
2 & & 0.898 & 0.545 \\
3 & & 0.870 & 0.423 \\
\hline 1 & $24-24.5$ & 0.304 & 0.126 \\
2 & & 0.640 & 0.269 \\
3 & & 0.433 & 0.118 \\
\hline 1 & $24.5-25$ & 0.132 & 0.073 \\
2 & & 0.488 & 0.122 \\
3 & & 0.338 & 0.076 \\
\hline
\end{tabular}

\subsection{Photometric completeness}

We used the ADDSTAR routine in DAOPHOT to determine the completeness of our photometry. We tested each frame separately in both the $J$ and $H$ band, by adding about $10 \%$ of the total number of detected stars in order not to increase the crowding in the images. We performed four runs for several magnitude bins. The results are shown in Table 3 for all the observed fields. Since NIC3 is less sensitive in the $H$ band, the completeness in $H$ significantly affects our subsequent data analysis. In the case of Field 1, for which only short exposures are available, the completeness rapidly decreases to $\sim 50 \%$ at $H \simeq 23$. Fields 2 and 3, on the other hand, reach a completeness of $\sim 50 \%$ and $\sim 40 \%$, respectively, at $H \simeq 24$.

\section{Analysis of the NICMOS data}

\subsection{The colour-magnitude diagram}

We have corrected the observed magnitudes of our sample for reddening by assuming $E(B-V)=0.1$ from Durrell \& Harris (1993). This implies $A_{J}=0.1$ and $A_{H}=0.06$.

The de-reddened colour-magnitude diagram (CMD) is plotted for each of the three fields and for the whole sample in Fig. 1. For the stars in common to more than one field, we have adopted the mean magnitudes and colours computed as above. Similar levels of photometric completeness are reached for all three fields at magnitudes brighter than $H_{0} \simeq 23$, so that their CMDs can be directly compared to one another. In all the three cases the main sequence is well defined down to $H_{0} \simeq 22$ and spreads out for $-0.4<(J-H)_{0}<1.8$ at fainter magnitudes due to our photometric uncertainty. The 

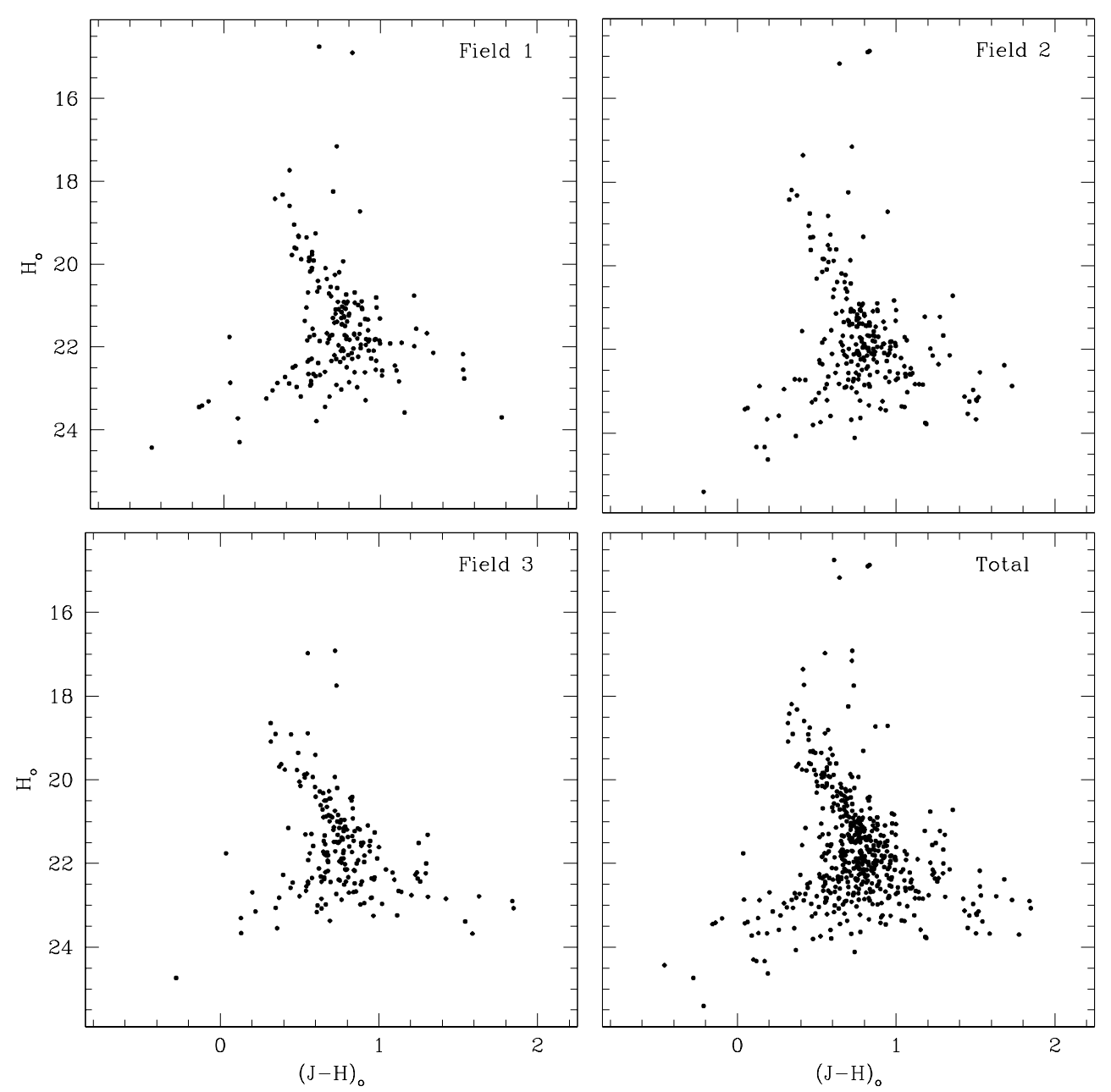

Fig. 1. De-reddened CMD of each observed field and all of the fields combined. Stars common to two fields have been removed from each field but are included in the CMD of the whole sample, with averaged magnitudes and colours.

few stars brighter than $H_{0}=18$ are probably foreground objects. Indeed, Durrell \& Harris (1993) determined the turnoff for $\mathrm{M} 15$ at $V_{0}=19.4$ which corresponds to $H_{0}=18.4$ from the theoretical track of Baraffe et al. (1997) at the metallicity of M $15([\mathrm{Fe} / \mathrm{H}]=-2.15)$. This implies that the stars at $H_{0} \simeq 18$ are either cluster objects evolved off the main sequence or simply foreground stars.

The bright tip of the main sequence shows up at $H_{0} \simeq 18$ for Fields 1 and 2 while it falls at $H_{0} \simeq 19$ for Field 3. This apparently fainter turn-off magnitude is likely due to statistical fluctuations in our small sample.

In order to reduce the contamination from foreground and background stars, we applied to the CMD of each field a $2.5 \sigma$ clipping selection around the average colour of the main sequence. The resulting three decontaminated samples were merged onto the CMD of Fig. 2 where the stars in common are represented with averaged magnitudes and colours. Using the predictions of Ratnatunga \& Bahcall (1985), we have estimated the field-star contamination to be about 4 stars for each NICMOS field in the direction of M 15. This estimate is valid for a limiting magnitude of $H=23$ (i.e. $0.2 M_{\odot}$ in Fig. 2), which corresponds to $V \simeq 27$ in the evolutionary tracks of Baraffe et al. (1997), and is integrated over the whole $(B-V)$ colour range taken into account by Ratnatunga $\&$ Bahcall (1985). Therefore, we do not expect the CMD to change significantly after the $\sigma$-clipping is applied. The photometric errors are also indicated on the left-hand side of Fig. 2: in the range $14 \leq H_{0} \leq 22$ the $(J-H)_{0}$ colours are known with an accuracy of $\pm 0.07 \mathrm{mag}$, at $22 \leq H_{0} \leq 23$ they increase to $\pm 0.13 \mathrm{mag}$ and for $H_{0}>23$ the photometric accuracy is as poor as $\pm 0.24 \mathrm{mag}$.

Superposed on the observed CMD distribution is the theoretical track obtained from the models of Baraffe et al. (1997) for a metallicity of $[\mathrm{M} / \mathrm{H}]=-2$, which closely matches the $[\mathrm{Fe} / \mathrm{H}]=-2.15$ value of $\mathrm{M} 15$. This track is scaled by the distance modulus of $15.11 \mathrm{mag}$ (Durrell \& Harris 1993). The stellar masses actually defining the theoretical track are listed on the right-hand side of Fig. 2 for decreasing $H_{0}$ magnitudes. The main sequence spans a mass range between $0.8 M_{\odot}$ and $0.2 M_{\odot}$.

\subsection{The luminosity function}

The LF observed for the external fields of M 15 is plotted in Fig. 3 (solid line) corrected for incompleteness. The stars in the CMD were grouped into magnitude bins (each 0.5 mag wide) 


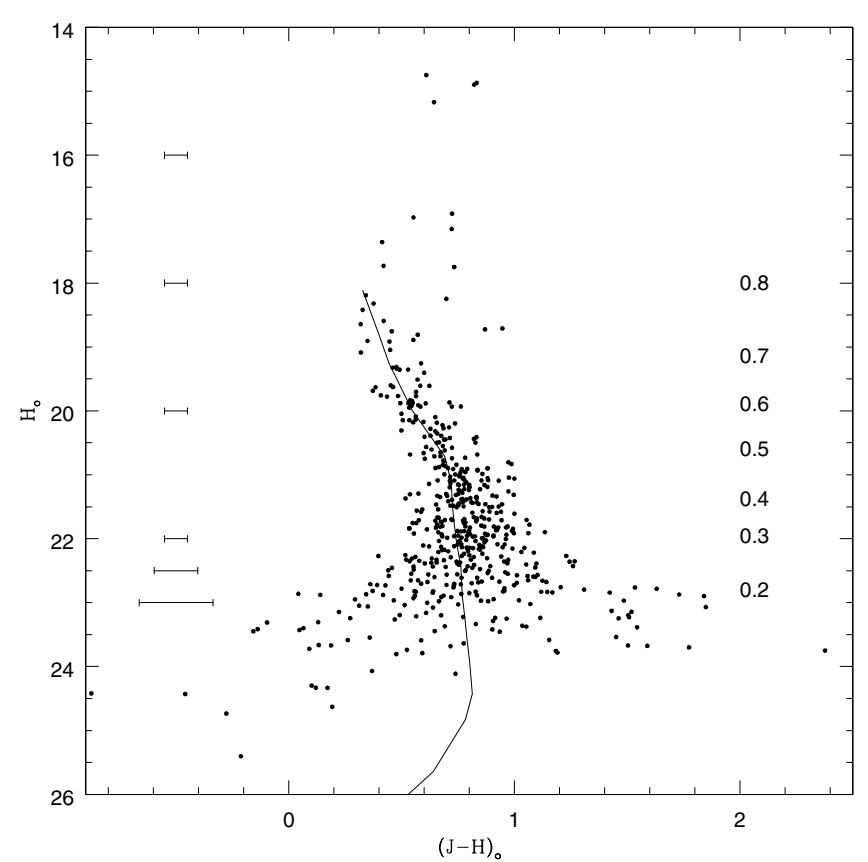

Fig. 2. De-reddened CMD of the whole sample on which the theoretical track of Baraffe et al. (1997), computed for the metallicity of M 15 $([\mathrm{Fe} / \mathrm{H}]=-2.15)$, has been superposed. Photometric errors are represented, as a function of $H_{0}$, at the left-hand side of the diagram, whilst the mass range spanned by main sequence stars is marked on the right-hand side of the plot, in unit of solar masses.

between $H_{0}=18$ and $H_{0}=24$, where completeness drops to less than $50 \%$. Since the completeness of the three fields is nearly the same at $H_{0}<23$, we have assumed a mean completeness factor with which we have corrected the star counts of bins brighter than 23. At $H_{0}>23$, Fields 2 and 3 are the major contributors to the observed LF, since they are deeper. In this magnitude range we have, therefore, ignored the stars in Field 1 not contained in Fields 2 or 3 and rescaled the total number of objects in these two latter fields to match the total area of the survey. Therefore we computed a mean completeness factor between these two fields and used the resulting value to correct the star counts of bins fainter than $H_{0}=23$. Given the shallower photometric depth of Field 1, having ignored its contribution to the LF for $H_{0}>23$ is not likely to affect the statistical significance of our results.

The LF of Fig. 3 extends over the range $2 \leq M_{H} \leq 9$, peaking at $M_{H}=7.1$ (corresponding to $M_{I}=8.5$; Baraffe et al. 1997) and is characterised by a quite sharp drop to fainter magnitudes due to a lack of progressively less massive stars. Moreover, this LF confirms the general behaviour found by Paresce \& De Marchi (2000) for a dozen Galactic globular clusters: their average LF, computed from stars below $1 M_{\odot}$ near the half-light radius, rises to a maximum value at $M_{I} \simeq 8.5-9$ and then drops for fainter magnitudes (this applies regardless of the cluster position and orbit in the Galaxy and of its internal dynamical state).

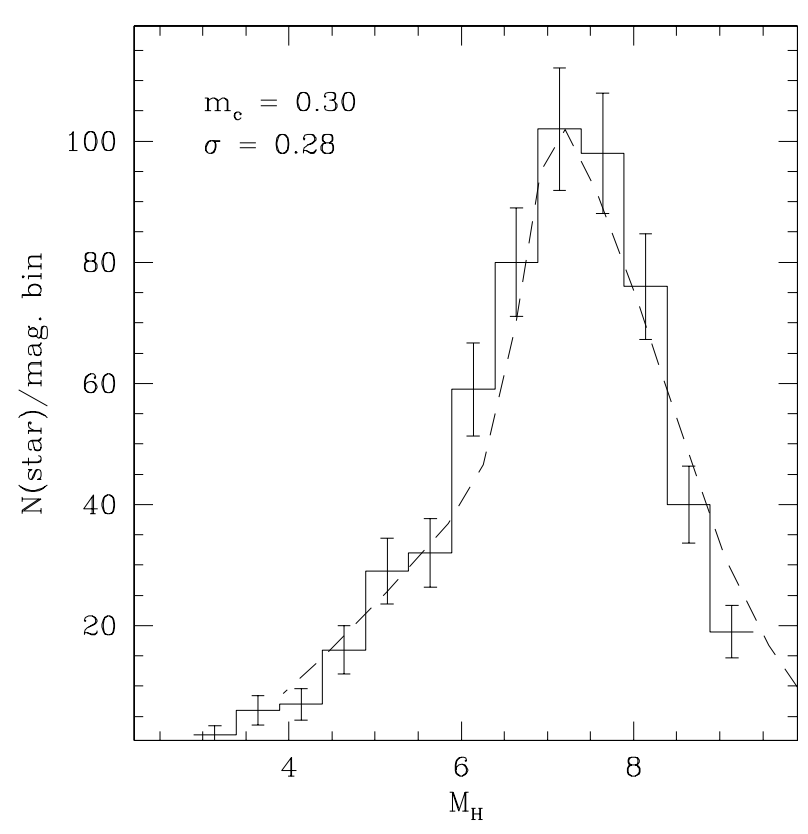

Fig. 3. Luminosity function (solid curve) of the whole sample, corrected for photometric completeness. Poissonan errors have been associated to the observed star counts. The dashed distribution is the best fitting LF, computed under the assumption of a log-normal mass distribution with a characteristic mass of $0.3 M_{\odot}$.

\subsection{The mass function}

The MF of the observed fields was derived from the LF of Fig. 3. Instead of deriving the MF by inverting the LF, we followed the approach of Paresce \& De Marchi (2000), so as to treat separately observational and theoretical uncertainties. We assumed a model MF of log-normal type, i.e. one of the type:

$\ln \left(\frac{\mathrm{d} N}{\mathrm{~d} \log (m)}\right)=A-\left[\frac{\log \left(m / m_{\mathrm{c}}\right)}{\sqrt{2} \sigma}\right]^{2}$

with characteristic mass $m_{\mathrm{c}}$ and standard deviation $\sigma . A$ is a normalization constant. We then folded it through the derivative of the mass-luminosity relationship of Baraffe et al. (1997) to obtain a model LF, which we compared to the data until a suitable value of the parameters was found that gives a good fit to the observations. The best fitting LF is superposed to the observations in Fig. 3 as a dashed distribution and is obtained with $m_{\mathrm{c}}=0.3 M_{\odot}$ and $\sigma=0.28$.

\section{A comparison with existing surveys of $M 15$}

As mentioned in the Introduction, M 15 has been extensively studied for it is at a relatively large distance from the Galactic plane and, consequently, not severely affected by dynamical interactions with the Galaxy.

Figure 4 spatially visualises the stellar fields observed in the core of M 15 by De Marchi \& Paresce (1996; DMP96) and Sosin \& King (1997; SK97-1, SK97-2) in order to derive the central MF. The DMP96 and SK97-1 fields are at the centre, whilst the SK97-2 field is 20" away from it. All fields are well within the half-light radius of $\mathrm{M} 15\left(r_{h} \simeq 1^{\prime}\right.$; Trager et al. 1995). Both studies detect a substantial amount of 


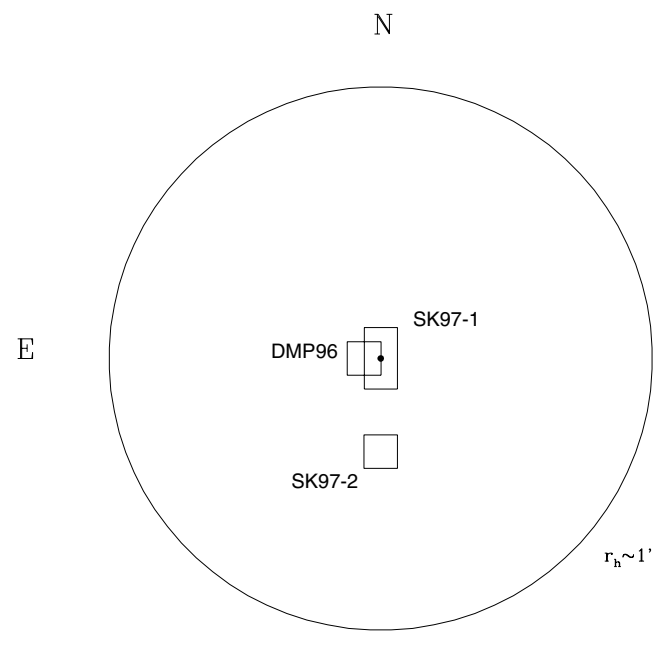

Fig. 4. Spatial distribution of the stellar fields observed within the halflight radius of M 15 (1'; Trager et al. 1995). DMP96 is the field studied by De Marchi \& Paresce (1996) near the core, while SK97-1 and SK97-2 are the areas surveyed by Sosin \& King (1997). SK97-2 is $20^{\prime \prime}$ away from the centre.

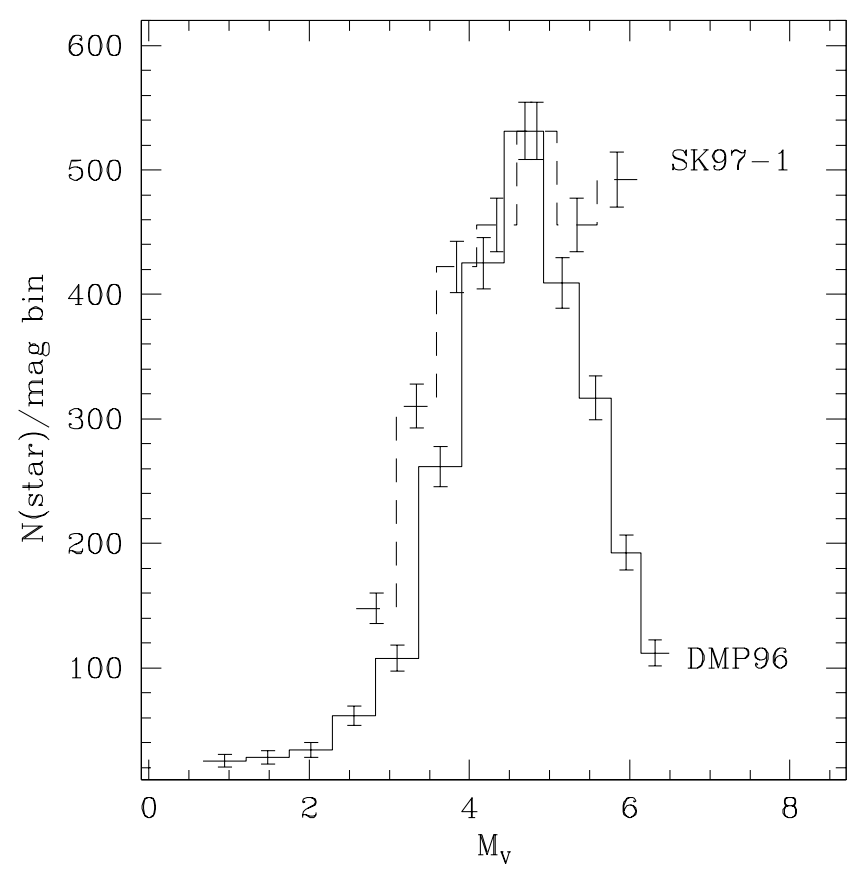

Fig. 5. Luminosity functions as derived by De Marchi \& Paresce (1996; solid curve) and Sosin \& King (1997; dashed distribution) for their field positioned at the centre of M 15. Poisson errors have been associated with the plotted star counts.

mass segregation, although the LFs differ for $M_{V}>4.8$. The two luminosity distributions are plotted in Fig. 5: the LF of De Marchi \& Paresce (1996) has been here translated from the original FOC F346M band magnitudes to standard Johnson $V$ values using Baraffe et al.'s tracks (private communication).

Both LFs have been normalised to the peak star count. An interesting point is that at $M_{V}>4.8$ the LF of De Marchi \& Paresce (1996) dramatically drops, whilst the LF derived by Sosin \& King (1997) is flat. We believe that this discrepancy is
Table 4. M 15 surveys.

\begin{tabular}{lcc}
\hline \hline & $\begin{array}{c}\text { Distance from } \\
\text { the center }\end{array}$ & Filters \\
\hline Durrell \& Harris (1993) & $7^{\prime} \mathrm{NW}$ & $B, V$ \\
De Marchi \& Paresce (1995) & $4^{\prime} \cdot 6 \mathrm{NW}$ & $V, I$ \\
This work & $7^{\prime} \mathrm{NE}$ & $J, H$ \\
\hline
\end{tabular}

$\mathrm{N}$

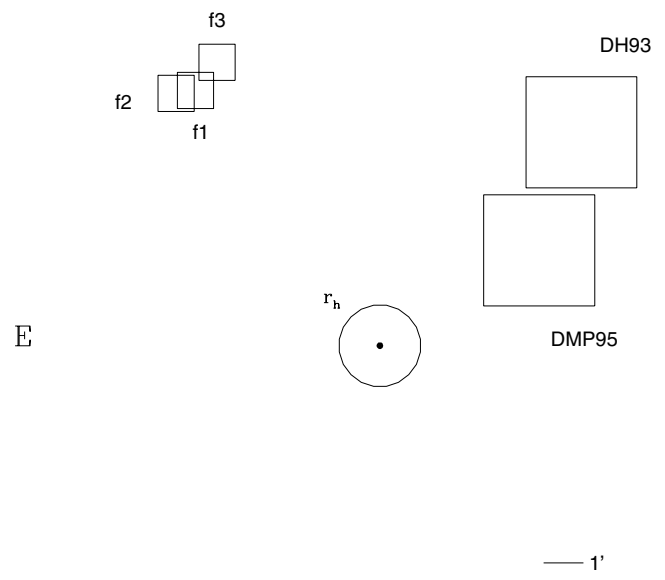

Fig. 6. Spatial distribution of the fields observed at distance larger than the cluster half-light radius. DH93 labels the field imaged by Durrell \& Harris (1993) at 7' NW, whilst DMP95 represents the field observed by De Marchi \& Paresce (1995) at 4'.6 NW from the centre. f1, f2 and $\mathrm{f} 3$ are the NIC3 fields analysed in this paper.

due to photometric incompleteness and to the colour extrapolation from the UV to the $V$ band.

Whilst mass segregation affects the innermost region of globular clusters, dynamical interactions with the Galaxy act predominantly on their outermost regions inducing stellar evaporation and stripping. Thus, clusters as rich as M 15 are likely to be dynamically unperturbed at their half-light radius (Richer et al. 1991) and stars at this distance can be used to constrain the IMF. Table 4 lists the observations available in the literature which cover the outskirts of M 15. Their corresponding fields are plotted in Fig. 6 together with the cluster centre and half-light radius.

We extracted the LFs from these papers and homogenised them in units of I magnitudes by using Baraffe et al.'s (1997) tracks. In Fig. 7 we over-plot the LF derived from the NIC3 data on Durrell \& Harris' (1993; left-hand panel) and De Marchi \& Paresce's (1995; right-hand panel) distributions. The latter were scaled to the peak star counts of the NIC3 distribution. Unfortunately, Durrell \& Harris' (1993) observations are not deep enough to reach the LF turn-over at $M_{I} \simeq 8.5$. Nevertheless, their LF agrees well with the ascending part of the luminosity distribution derived from the NIC3 exposures. Very good overlap is instead achieved between the LFs of this paper and that of De Marchi \& Paresce (1995) over 

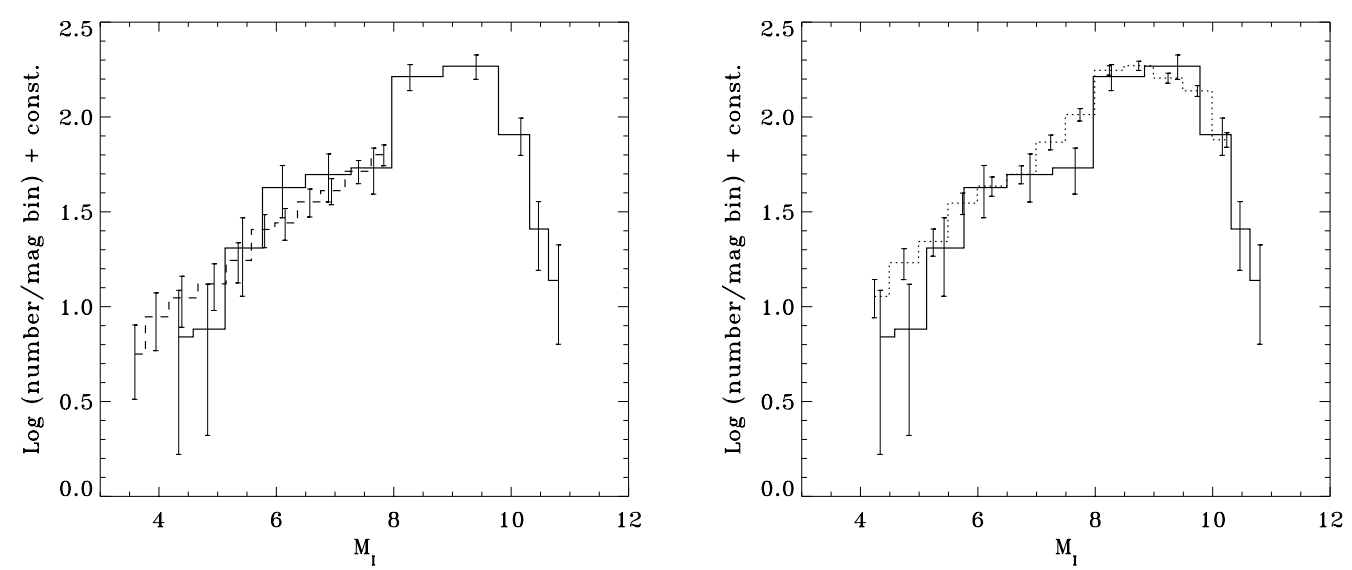

Fig. 7. The LFs computed by Durrell \& Harris (1993, DH93) and De Marchi \& Paresce (1995, DMP95) are compared with the distribution obtained from the NIC3 data. All of the luminosity distributions have been translated into $\mathrm{M}_{I}$ values and scaled to the peak star-counts of the NIC3 luminosity function.

the range $4 \leq M_{I} \leq 10$, so that both resulting MFs peak near $0.3 M_{\odot}$.

\section{Dynamical structure}

Having derived the spatially resolved LF for M 15, we can now study its radial changes and address the issue as to whether they are consistent with mass segregation ensuing from two body relaxation. To study the dynamical properties of the cluster, we employed the multi-mass Michie-King models originally developed by Meylan (1987, 1988) and later suitably modified by Pulone et al. (1999) and De Marchi et al. (2000) for the general case of clusters with a set of radially varying LFs. Each model run is characterised by a MF in the form of a power-law $\mathrm{d} N / \mathrm{d} \log m \propto m^{-x}$, with a variable index $x$, and by four structural parameters describing, respectively, the scale radius $\left(r_{\mathrm{c}}\right)$, the scale velocity $\left(v_{\mathrm{s}}\right)$, the central value of the dimensionless gravitational potential $W_{\mathrm{o}}$, and the anisotropy radius $\left(r_{\mathrm{a}}\right)$. (After having suggested, in Sect. 3, a rather general description of the functional form of the MF, namely a log-normal distribution, it might seem inappropriate to adopt a variable power-law as the basis for the MF in our dynamical model. In fact, as we show below, the resulting MF is indistinguishable, for any practical purposes, from a log-normal distribution.)

From the parameter space defined in this way, we selected those models that simultaneously fit both the observed surface brightness profile (SBP) and velocity dispersion profile (VDP) of the cluster as measured, respectively, by Guhathakurta et al. (1996; for $\left.r<100^{\prime \prime}\right)$ and Trager et al. (1995; for $\left.r>100^{\prime \prime}\right)$ and by Gebhardt et al. (2000). However, even requiring good fits to both the SBP and VDP can, by itself, only constrain $r_{\mathrm{c}}, v_{\mathrm{s}}, W_{\mathrm{o}}$, and $r_{\mathrm{a}}$, whilst still allowing the MF to take on a variety of shapes. To break this degeneracy, we further imposed the condition that the model MF agree with the observed LF at all radial distances offered by the data.

Since Michie-King modeling only provides a "snapshot" of the current dynamical state of the cluster, it is useful to refer to the GMF, i.e. the mass distribution of all cluster stars at present, or, in other words, the MF that the cluster would have simply as a result of stellar evolution (i.e. ignoring any local modifications induced by internal dynamics and/or the interaction with the Galactic tidal field). Clearly, in this case the IMF and GMF of main sequence (unevolved) stars is the same. For practical purposes, the GMF has been divided into sixteen different mass classes, covering main sequence stars, white dwarfs and heavy remnants, precisely as described in Pulone et al. (1999).

Our parametric modelling approach assumes energy equipartition amongst stars of different masses. Thus, we ran a large number of trials to see whether we could find a set of parameters for the GMF (i.e. a suitable GMF "shape") such that the local MFs produced by mass segregation would locally fit the observations. We note here that, rather than converting the observed LFs into MFs for comparison with the predictions of the model, we prefer to keep observational errors and theoretical uncertainties separate. Therefore, we convert the model MFs to LFs using for all the same $M-L$ relation, namely that of Baraffe et al. (1997), precisely as we did in Fig. 3. Not surprisingly, our exercise confirms what we had already shown in that figure and described above: as long as a single value of the index $x$ is used for the GMF over the mass range $0.2-0.8 M_{\odot}$, none of the predicted local LFs can be fitted to our data. In fact, a change of slope is needed at $m \simeq 0.3 M_{\odot}$ so that both the rising and dropping portions of the local LF can be simultaneously reproduced. If we then allow the MF to take on more than one slope, the GMF that best fits the observations is one with $x=0.9$ for stars in the range $0.3-0.8 M_{\odot}$ and $x=-2.2$ at smaller masses. The shape of this GMF is, thus, very similar to the log-normal distribution shown in Fig. 3.

The set of LFs predicted by the set of Michie-King parameters that best fit all available observations is shown in Fig. 8, where the squares correspond to the LFs available in the literature for this cluster at various distances from its centre. The fit to the SBP and VDP obtained with the same set of parameter values is shown in Figs. 9 and 10 and is surprisingly good. The values of the best fitting structural parameters are shown in Table 5, where they can be compared with those in the literature. The agreement is excellent, apart from a small difference in the value of the tidal radius which is, admittedly, not 


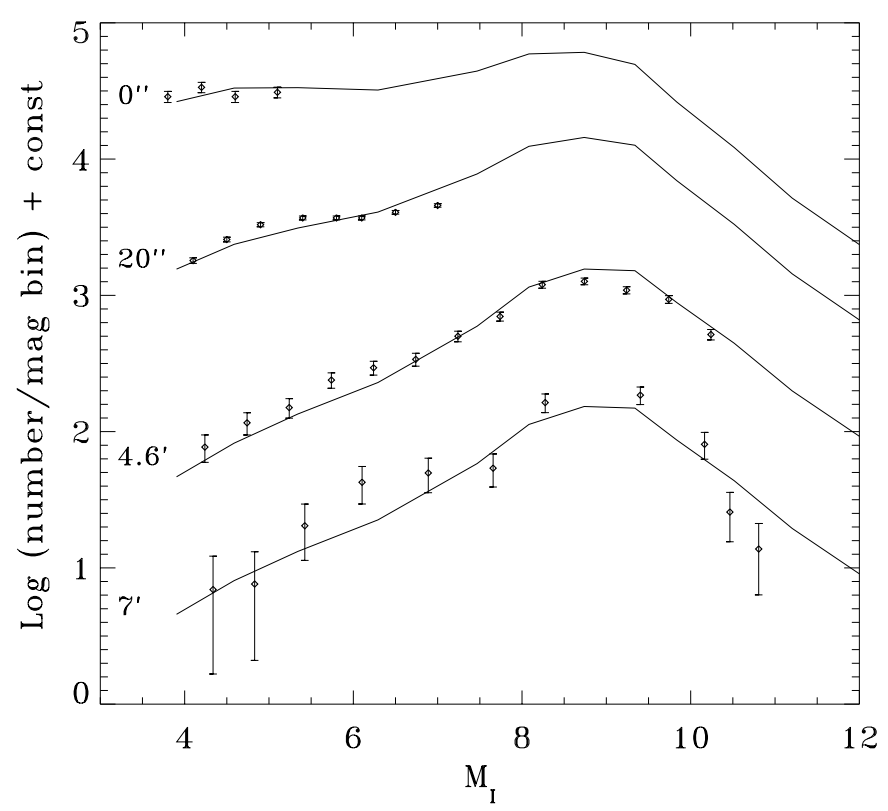

Fig. 8. The best fits of the Michie-King models to the LF observed at 7' (this work), 4.6 (De Marchi \& Paresce 1995), and 20" (Sosin \& King 1997) from the cluster centre and in the core (De Marchi \& Paresce 1996; Sosin \& King 1997).

seriously constrained by our data. We note here that we can directly compare the observed SBP with our model since the solid line in Fig. 9 corresponds to stars of $\sim 0.8 M_{\odot}$, namely those contributing most of the cluster's light. As one should expect, stars in different mass classes have different projected radial distributions.

Although stars more massive than $\sim 0.8 M_{\odot}$ have evolved and are no longer visible, the shape of the IMF in this mass range has strong implications on the fraction of heavy remnants in the cluster and, as such, on the central velocity dispersion. We find that the best fit to the data and to the cluster's structural parameters, as given above, requires a value of $x=0.8$ for stars in the range $100-0.8 M_{\odot}$. It should be noted that the global cluster MF is thus slightly shallower than Salpeter's IMF, which would have $x=1.35$. The total implied cluster mass is $\sim 5.4 \times 10^{5} M_{\odot}$ and the mass-to-light ratio is on average $M / L \simeq 2.1$, with $M / L \simeq 3.7$ in the core. The total cluster luminosity $L_{V}$ has been estimated by integrating the best-fitting SBP (solid line in Fig. 9), properly normalised to match the observed central surface brightness. The best fitting models suggest that a large fraction of mass (of order $\sim 4 \%$ ) is trapped in heavy remnants, namely stellar black holes, neutron stars and white dwarfs. However, the presence of an intermediate mass black hole is not required.

Interestingly, the rather shallow GMF that we obtain for stars above $0.8 M_{\odot}$, which, in turn, results in a large fraction of heavy remnants, is also dictated by the central enhancement seen in the SBP. The excellent fit that our model offers to the radial surface density of TO-mass stars (Fig. 9) would not be otherwise possible. A steeper MF index would result in a shallower central profile which would fail to reproduce the central density enhancement. It should also be noted that, since a canonical King-type profile does not reproduce the SBP

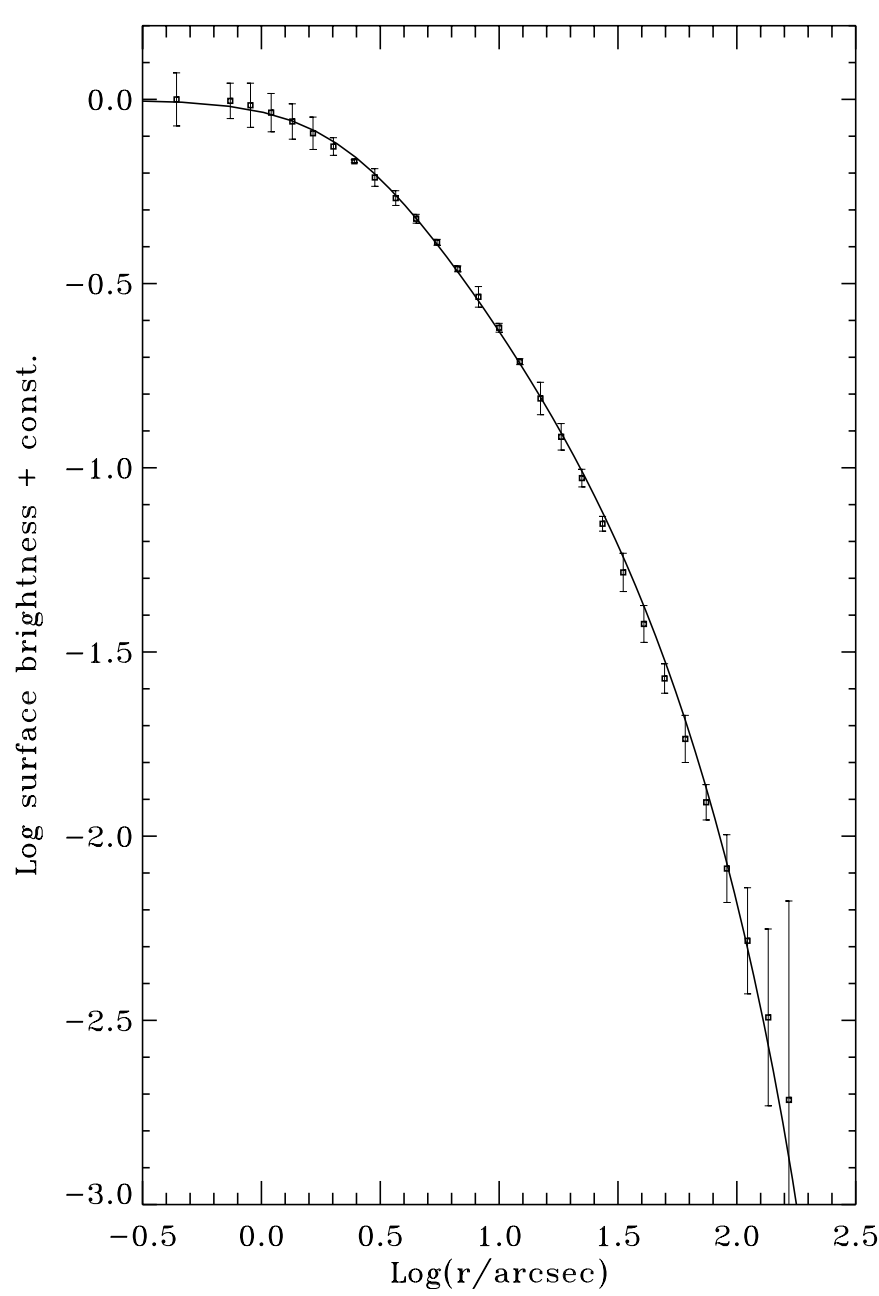

Fig. 9. Model fit to the surface brightness profile. The solid line corresponds to the profile of stars of $\sim 0.8 M_{\odot}$, responsible for most of the light of the cluster.

of M 15 (see, e.g., Trager et al. 1995), the value of $r_{\mathrm{c}}$ that we obtain does not correspond with the canonical definition of core radius, namely that at which the surface density falls to one-half its central value. The latter definition only applies to the profile of the original King model with a single mass component (see Kent \& Gunn 1982). In our case, $r_{\mathrm{c}}$ simply represents a scale radius.

\section{Other Michie-King models for M 15}

Contrary to what we have concluded here, in their analysis of the radial variation of the LF of M 15, Sosin \& King (1997) concluded that a multi-mass Michie-King model is unable to reproduced the observations. As their Fig. 12 shows, the variation predicted by their multi-mass model is larger than that observed when comparing the centre of the cluster and the region at $r \simeq 5^{\prime}$. We have identified three reasons that might have led Sosin \& King (1997) to this conclusion and we discuss them here briefly.

The first is mostly related to the approach used and, as such, should only affect the uncertainty of the results. Sosin \& King (1997) decided to transform the observed LFs into MFs, 


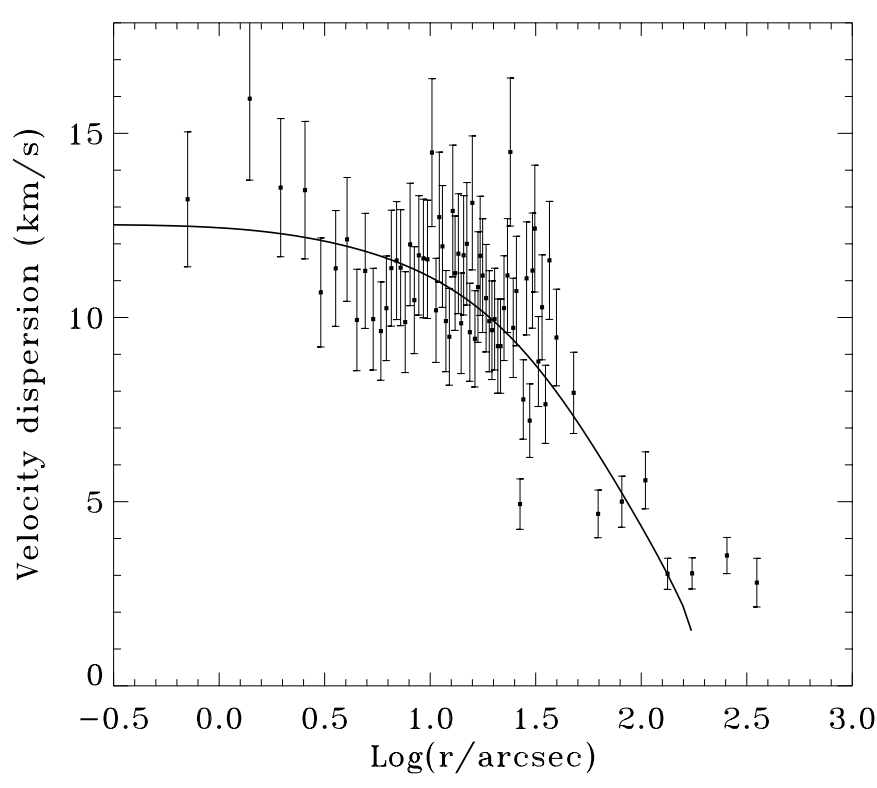

Fig. 10. Model fit to the velocity dispersion profile.

not necessarily using the same $M-L$ relation for all data, and to compare the predictions of their multi-mass models to these MFs. The advantage of our approach, in which the LF predicted by the model is compared with the observed LF, is that we ensure that observational errors and theoretical uncertainty (in the model and $M-L$ relation) are kept separate and that only one $M-L$ relation is used throughout the process.

Secondly, it appears that their dynamical model is unable to reproduce at all the observed velocity dispersion profile, as the authors themselves point out. Conversely, ours is in excellent agreement with the observations. As a result, Sosin \& King (1997) predict a fraction of heavy remnants $(\leq 1 \%)$ well below the current estimates for this cluster.

Most importantly, however, we believe that their inability to reproduce the observed radial variation of the LF stems from the functional form of the MF that they adopt. As we describe in Sect. 4, we have made a general assumption about the shape of the GMF, in the form of a log-normal distribution, based on what was learnt from the observation of a large number of halo GCs (Paresce \& De Marchi 2000). We then let our procedure find the parameter values that simultaneously fit all available data. Since the number of independent measurements is larger than that of the unknowns, the procedure is bound to converge. On the other hand, Sosin \& King (1997) adopt the MF determined by Piotto et al. (1996) at $r \simeq 5^{\prime}$ as the basis for their dynamical model, but the MF predicted by it for the cluster core fails to match the data. The origin of the mismatch seems to lie predominantly in the assumed shape of the MF, which is flat in the range $m \geq 0.7 M_{\odot}$ and then sharply rises at lower masses. This results in a MF in the central cluster regions that sharply drops in the range $0.8 M_{\odot}>m>0.7 M_{\odot}$. It appears that having adopted a model MF with a more gentle rise from $0.8 M_{\odot}$ all the way through to $0.5 M_{\odot}$ (which still fits the data at $5^{\prime}$ equally well) would have produced a MF in considerably better agreement with the observations in the central cluster region.
Table 5. Parameters of the Michie-King models used for M 15.

\begin{tabular}{lccc}
\hline \hline Parameter & $\begin{array}{c}\text { Fitted } \\
\text { value }\end{array}$ & $\begin{array}{c}\text { Literature } \\
\text { value }\end{array}$ & Ref. \\
\hline Core radius $r_{\mathrm{c}}$ & $2{ }^{\prime \prime} 3$ & $2^{\prime \prime}$ & $a$ \\
Tidal radius $r_{\mathrm{t}}$ & $17^{\prime}$ & $21{ }^{\prime}$ & $b$ \\
Half-light radius $r_{\mathrm{h}}$ & 1.3 & $1^{\prime}$ & $c$ \\
Central vel. disp. $\sigma_{\mathrm{v}}$ & $12.1 \mathrm{~km} \mathrm{~s}^{-1}$ & $11.7 \mathrm{~km} \mathrm{~s}^{-1}$ & $d$ \\
\hline
\end{tabular}

$a$ : Guhathakurta et al. (1996).

$b$ : Harris (1996).

$c$ : Djorgovski (1993).

$d$ : Gebhardt (2000).

\section{Conclusions}

We have analysed HST/NICMOS parallel data available for three fields in the Galactic globular cluster M 15 located at $7^{\prime} \mathrm{NE}$ of the cluster centre. Their total LF extends over the range $2 \leq M_{H} \leq 9$ and peaks at $M_{H}=7.1$ (or $M_{I} \simeq 8.5$ ). It also shows a sharp drop towards fainter magnitudes, which is a typical signature of the lack of progressively less massive stars. We have fitted this LF with a log-normal mass distribution and obtained a characteristic mass of $0.3 M_{\odot}$, with a standard deviation of 0.28 . These values agree well with the characteristic mass and standard deviation derived for a dozen Galactic globular clusters by Paresce \& De Marchi (2000), thus supporting the ubiquity of the log-normal mass distribution for globular clusters.

The $H$-band LF obtained at $7^{\prime}$ from the centre of M 15 has been compared with the LFs derived by Durrell \& Harris (1993) and De Marchi \& Paresce (1995) at 7' NW and 4'.6 NW of the cluster centre, respectively. The comparison has required the translation of all original observed magnitudes into the $I$ band. The overlap among these three LFs is excellent, indicating that the distribution of the stars at distances larger than the half-light radius $\left(\sim 1^{\prime}\right.$; Trager et al. 1995$)$ may not be significantly perturbed in M 15 , as expected from its Galactocentric distance $\left(R_{\mathrm{G}} \simeq 11 \mathrm{Kpc}\right.$; Gnedin \& Ostriker 1997) and the small ellipticity of its orbit ( $e=0.32$; Dinescu et al. 1999). Indeed, calculations by Gnedin \& Ostriker (1997) show that the time to destruction of M 15, due to the combined effects of internal dynamical evolution and interaction with the Galaxy, is as large as 50 Gyr. Therefore, if correct, this time-scale would suggest that the outermost fields observed in M 15 have not been significantly perturbed by tidal stripping and evaporation and that their content should likely represent the initial stellar mass distribution. In other words, the high degree of similarity between the LFs (and hence the MFs) of the fields at 4.'6 and 7' from the centre would imply that these are very close to be the cluster IMF and there are no significant radial variations in the cluster IMF.

We have used the LF measured for M 15 at $7^{\prime}$ (this work), 4.6 (De Marchi \& Paresce 1995), and 20" (Sosin \& King 1997) from the cluster centre and in the core (De Marchi \& Paresce 1996; Sosin \& King 1997) to study the effects of mass segregation. We have fitted Michie-King models to the observed 
surface brightness and velocity dispersion profiles in order to estimate the cluster structural parameters and to the observed LF to constrain the shape of the cluster GMF. The latter turns out to be characterised by two slopes, $x=0.9$ for stars in the range $0.3-0.8 M_{\odot}$ and $x=-2.2$ at smaller masses, and is thus very close to the log-normal distribution obtained directly from our NIC3 data near the cluster's half-light radius.

The values of the cluster structural parameters that best fit the observations imply a cluster total mass of $\sim 5.4 \times 10^{5} M_{\odot}$ and a mass-to-light ratio of $M / L \simeq 2.1$ on average, with $M / L \simeq$ 3.7 in the cluster core. In addition, the best-fitting Michie-King model parameters suggest a slope of $x=0.8$ for the IMF in the range $100-0.8 M_{\odot}$, which supports the presence of a large fraction of heavy remnants ( 44\%) in the core. If such a high fraction of heavy remnants is present, as originally suggested by Illingworth \& King (1977), it would rule out the need for an intermediate-mass black hole to explain the mass segregation and velocity dispersions observed in the core of M 15.

Acknowledgements. We are very grateful to Carlton Pryor, the referee of this paper, for comments that have substantially strengthened the presentation of our work. It is a pleasure to thank Isabelle Baraffe and France Allard for providing us the theoretical tracks for the HST/FOC filters F253M and F346M and Francesco Paresce for useful discussions. M.S.B. acknowledges support from the Osservatorio Astronomico di Cagliari and from the Director General's Discretionary Fund at ESO.

\section{References}

Bailyn, C. D., Grindlay, J. E., Cohn, H., et al. 1989, AJ, 98, 882

Baraffe, I., Chabrier, G., Allard, F., \& Hauschildt, P. 1997, A\&A, 327, 1054

Baumgardt, H., Hut, P., Makino, J., et al. 2003, ApJ, 582, 21

Cederbloom, S. E., Moss, M. J., Cohn, H. N., et al. 1992, AJ, 103, 480
Dauphole, B., Geffert, M., Colin, J., et al. 1996, A\&A, 313, 119

De Marchi, G., \& Paresce, F. 1994, ApJ, 422, 597

De Marchi, G., \& Paresce, F. 1995, A\&A, 304, 202

De Marchi, G., \& Paresce, F. 1996, ApJ, 467, 658

De Marchi, G., Paresce, F., \& Pulone, L. 2000, ApJ, 530, 342

Dinescu, D. I., Girard, T. M., \& van Altena, W. F. 1999, AJ, 117, 1792

Djorgovski, S. 1993, in Structure and Dynamics of Globular Clusters, ed. S. Djorgovski, \& G. Meylan (San Francisco: ASP), ASP Conf. Ser., 50, 373

Djorgovski, S., \& King, I. R. 1986, ApJ, 305, L61

Durrell, P. R., \& Harris, W. E. 1993, AJ, 105, 1420

Gebhardt, K., Pryor, C., O’Connell, R., Williams, T., \& Hesser, J. E. 2000, AJ, 119, 1268

Gerssen, J., van der Marel, R. P., Gebhardt, K., et al. 2003, AJ, 125, 376

Gnedin, O. Y., \& Ostriker, J. P. 1997, ApJ, 474, 223

Guhathakurta, P., Yanny, B., Schneider, D. P., \& Bahcall, J. N. 1996, AJ, 111, 267

Harris, W. E. 1996, AJ, 112, 148

Illingworth, G., \& King, I. R. 1977, ApJ, 218, L109

Kent, S. M., \& Gunn, J. E. 1982, AJ, 87, 945

Krist, J., \& Hook, R. 1999, Tiny-Tim User Guide v5.0

Lauer, T. R., Holtzman, J. A., Faber, S. M., et al. 1994, ApJ, 369, L45

Meylan, G. 1987, A\&A, 184, 14

Meylan, G. 1988, A\&A, 191, 215

Paresce, F., \& De Marchi, G. 2000, ApJ, 534, 870

Peterson, R. C., Seitzer, P., \& Cudworth, K. M. 1989, ApJ, 347, 251

Piotto, G., Cool, A. M., \& King, I. R. 1997, AJ, 113, 1345

Pulone, L., De Marchi, G., \& Paresce, F. 1999, A\&A, 342, 440

Ratnatunga, K. U., \& Bahcall, J. N. 1985, ApJS, 59, 63

Richer, H. B., Fahlman, G. G., Buonanno, R., et al. 1991, ApJ, 381, 147

Sosin, C., \& King, I. R. 1997, AJ, 113, 1328

Storrs, A., Hook, R., Stiavelli, M., Hanley, C., \& Freudling, W. 1999, NICMOS ISR-99-005

Trager, S. C., King, I. R., \& Djorgovski, S. 1995, AJ, 109, 218 